symmetry with a three-fold rotation axis. All dipolar-like features (including the dipolar $\beta$ responses) sum to zero in such a system. So how does a finite $\beta$ remain? The answer is-far from intuitive, but this observation in TATB, and in the new molecule reported in this issue, has been rationalized by $\mathrm{Zyss}^{8}$ in terms of the octupolar response of the molecule (see part $b$ of the box).

By wrestling with group theory analysis Zyss showed that in general the $\beta$ tensor may contain contributions from odd-order multipoles. Thus in molecules of particular symmetries (for example trigonal or tetrahedral) significant octupolar contributions to the $\beta$ tensor can arise. The octupolar part of the $\beta$ tensor has many more independent components than the dipolar part (which vanishes anyway in octupolar molecules). When taken collectively, these components effectively pack more nonlinearity into a single molecule. Thus the use of octupolar geometries may allow a larger molecular nonlinearity per unit volume; a nonlinearity less sensitive to material orientation (a higher dimensionality of nonlinearity exists); and all without the possible detrimental effects of dipole moment.

The ruthenium complex reported in this issue clearly benefits from the additivity principle and has chirality built in to ensure octupolar crystallinity. The value of the off-resonance octupolar $\beta$ is indeed very large (in fact it exceeds the predicted tensor component sum by a factor of two) and the question now is how to make use of it. Crystals may show the highest bulk nonlinearity, but their processibility is limited; and Langmuir-Blodgett assembly is still not favoured by those looking for simple mass production. The preferred choice may be to incorporate the molecule into a polymer and perform some kind of 'poling'. Usually, this means dragging dipolar molecules into alignment by applying an electric field. In the absence of a dipole, conventional poling methods are ruled out, but collaborators of the CNET group may have the solution ${ }^{10}$. Termed 'orientational hole burning', the method relies on selective absorption by, and thus effective bleaching of, just those molecules in a specific orientational range, preventing total cancellation of the bulk nonlinearity. The orientational distribution of molecular $\beta \mathrm{s}$, now being asymmetric, allows for the bulk second-order effects.

Such an orientation scheme could be applied equally to polar molecules as to the new octupolar species. However, there must be question marks attached to the added complexity of the process and to the need for a resonant interaction with the material. Many of the more highly nonlinear molecules are also somewhat unstable to photo-induced degradation. The use of metal centres, as in the ruthenium complex, may ameliorate these problems, but by how much is unclear. The problem of 'locking in' the orientation is a further point to be addressed, although there might be useful parallels in the observation of photo-induced excitation and trans-to-cis isomerization seen in azo-dyes ${ }^{11,12}$

The key advance from which these developments have grown rests on the connection that Zyss made between the rather abstract areas of tensor analysis and group theory as applied to optical nonlinearities and the realization that molecules could be designed which show octupolar nonlinearities. The potential advantages are self-evident. What remains to be seen is whether other workers in the field are willing, or able, to move up from dipoles to octupoles in their search for improved nonlinear materials.

Graham Cross is in the Department of Physics, University of Durham, South Road, Durham DH1 3LE, UK.

1. Dhenaut. C. Ledoux, I. Samuel, I. D. W. Zyss, J. Bourgault, M. \& Le Bozec, H. Nature 374, 339-342 (1995).

. Marder, S. R. et al. Science 263, 511-514 (1994)

3. Laidlaw, W. M., Denning, R. G., Verbiest, T., Chauchard, E. \& Persoons, A. Nature 363.5860 (1993).

4. Szablewski, M. J. org. Chem. 59. 954-956 (1994).

5. Rao, V. P., Jen, A. K. Y., Wong, K. Y. \& Drost, K. J. J. chem. Soc chem. Commun. 1118 (1993)

6. Peterson, I. R. in Molecular Electronics (ed. Ashwell. G. J.) 117-206 (Wiley, Chichester, 1992).

7. Ledoux, I., Zyss, J., Siegel, J. S. \& Lehn, J.-M. Chem Phys. Lett. 172, 440-444 (1990)

8. Zyss, J. Nonlinear Optics 1, 3-18 (1991).

9. Zyss, J., Dhenaut, C., Chauvan, T. \& Ledoux, I. Chem Phys. Lett. 206. 409-414 (1993)

10. Nunzi, J.-M. Charra, F.. Fiorini, C. \& Zyss, J. J. chem Phys. Lett. 219. 349-354 (1994).

11. Jones, C. \&Day, S. Nature 351, 15 (1991).

12. Charra, F., Kajzar, F., Nunzi, J. M., Raimond, P. \& Idiart, E. Opt. Lett. 18, 941-943(1993)

\title{
From dipoles to octupoles
}

$a$

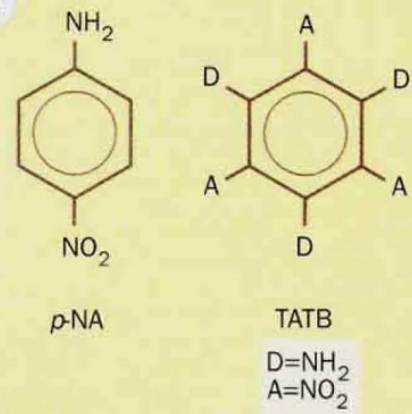

$b$

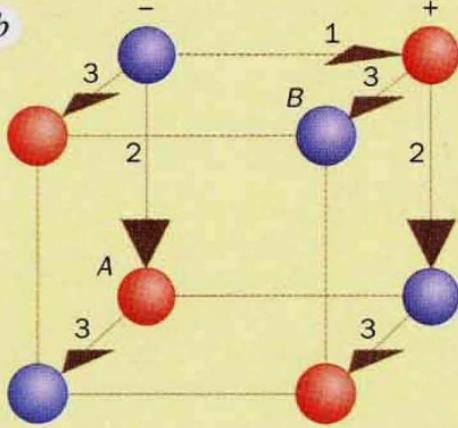

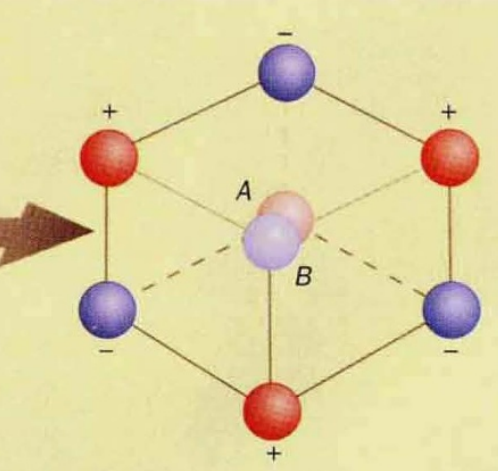

a, $p$-NA is a model chromophore for second-order nonlinear optics. The general scheme is donor; $\pi$-conjugated group; acceptor. The single dominant component of $\beta$ lies along the dipolar axis and its magnitude depends on the magnitude of $\Delta \mu$, the difference between ground and excited-state dipole moments. TATB is a trigonal symmetric version of $p$-NA having a complete net absence of dipole moment. The dipolar or vectorial part of the $\beta$ tensor vanishes but the symmetry is not high enough to prevent the presence of a finite octupolar $\beta$ tensor component. $b$, An octupole can be generated by successive translations and inversion of signs of point charges. Thus a dipole, more familiarly viewed as two equal and opposite charges separated by a certain distance, can be generated by a simple replication along a vector of a single point charge followed by a reversal of the sign of the new charge. After a further two operations of this type a cube is generated. This has $2^{n}$ ( $n$ being the number of operations) or 8 point charges and is thus an octupole. To see how this three-dimensional object maps onto the two-dimensional TATB molecule, one must project the cube along one of the body diagonals, whereupon $A$ and $B$ coincide and cancel. The octupole is a non-dipolar yet non-centrosymmetric structure and thus satisfies the requirements for a non-zero $\beta$ tensor. The full treatment of multipolar groups shows that structures possessing multipolar groups of higher order than $n=3$ will not possess a finite $\beta$. Interestingly, and indeed non-intuitively, this applies to structures with five-fold symmetry $(n=5)$ which are, nevertheless, noncentrosymmetric. 